\title{
Risk factors associated with methamphetamine use and heart failure among Native Hawaiians and other Pacific Island peoples
}

\author{
Marjorie K Mau' \\ Karynna Asao ${ }^{2}$ \\ Jimmy Efird ${ }^{3}$ \\ Erin Saito \\ Robert Ratner ${ }^{4}$ \\ Muhannad $\mathrm{Hafi}^{4}$ \\ Todd Seto 5 \\ 'Department of Native Hawaiian \\ Health; ${ }^{3}$ Biostatistics and Data \\ Management Facility; ${ }^{5}$ Department \\ of Medicine, John A. Burns School \\ of Medicine, University of Hawai'i at \\ Manoa; ${ }^{2}$ Claremont College; ${ }^{4}$ Medstar \\ Research Institute
}

\begin{abstract}
Objective: Heart failure (HF), a long term outcome of chronic methamphetamine use (MU), occurs more frequently in racial and ethnic minority populations at high risk for cardiovascular disparities. This study examined the association of socio-demographic and clinical risk factors with MU among heart failure patients who are Native Hawaiians (NH) or other Pacific Island peoples (PIP).

Design/Setting/Patient population: Cross-sectional study of NHs and PIPs with advanced heart failure enrolled in the Malama Pu'uwai Study, a randomized control trial to test an educational intervention to reduce re-hospitalization and/or death. A total of 82 participants were enrolled between 6/1/06 to 12/31/07 and met the following eligibility criteria: 1 ) self-identified NH or PIP, 2) Left ventricular systolic ejection fraction $\leq 45 \%, 3$ ) Age of 21 years or older. Data were analyzed by odds ratios (OR), $95 \%$ confidence intervals (CI), and multiple logistic regression analysis.
\end{abstract}

Main outcome measure: Methamphetamine use.

Results: Twenty-two percent of HF participants were identified as being current or prior methamphetamine users. Younger age and non-married status (combined never married or divorced/separated) were independently associated with MU after adjustment for sex, education, and other co-morbidities associated with $\mathrm{HF}$ (ie, age $>50$ years, $\mathrm{OR}=0.16,95 \% \mathrm{CI}, 0.03-0.84$; non-married status combined as never married $\mathrm{OR}=8.5, \mathrm{CI}, 1.5-47$; divorced/separated $\mathrm{OR}=11$, CI 1.8-75).

Conclusions: Risk factors associated with MU in NH and PIPs with heart failure include: younger age and being divorced/separated or never married. Health care providers should be aware of MU as a contributing factor in the approach and treatment of HF in NHs and PIPs.

Keywords: Native Hawaiian and other Pacific Islander, ethnic minority, methamphetamine use, heart failure

\section{Introduction}

Methamphetamine use (MU), initially recognized as a health problem limited to Hawai' $i$ and the western region of the US, has continued to spread eastward and has increasingly affected both rural and urban areas throughout the US. ${ }^{1}$ According to a 2005 national survey, an estimated 10.4 million people age 12 years or older have tried the highly addictive drug in the US. ${ }^{2}$ Minority populations are at higher risk, particularly racial/ethnic groups such as Native Hawaiians (NHs) and Pacific Island Peoples (eg, Samoan, Tongan, Micronesian) who are concentrated in the western regions of the US Native Hawaiians experienced a 10-fold increase in methamphetamine use between 1995-1998. ${ }^{3,4}$ In parallel with this upward trend, 5.6\% of all Native Hawaiian 12th graders in the state of Hawai'i have used methamphetamine at least
Correspondence: Marjorie K Mau 677 Ala Moana Blvd, Suite 1016-B; Honolulu, HI 968I3, USA

Tel + I 8085878557

Fax +l 8085878565

Emailmmau@hawaii.edu 
once in their lifetime compared with $4.8 \%$ among high school seniors nationally. ${ }^{1,5}$ In the wake of the growing epidemic of MU, health care providers and acute care hospitals are seeing long term health effects of methamphetamine use. $^{6-9}$ Between 2004 and 2005, estimated emergency department visits for methamphetamine abuse rose by almost $50 \%$ to 108,905 visits per year. ${ }^{10,11}$ In addition, treatment admissions for methamphetamine abuse also have increased to 150,000 admissions per year in 2004 and the number of states reporting high rates of admissions (defined as $>24$ per 100,000 population) increased from 5 states in 1992 to 21 states in $2002 .^{1}$

Despite the growing and persistent problem of MU, few studies have examined long term health problems attributable to chronic MU. Although behavioral and neurological effects of chronic MU have been reported, an emerging literature on the impact of chronic MU on the cardiovascular system has resulted in a growing recognition of methamphetamineassociated cardiomyopathy and heart failure. ${ }^{6-9,12}$ Heart failure is a major public health problem in the US with an estimated prevalence of 5.2 million and direct and indirect costs totaling $\$ 33.2$ billion. ${ }^{13}$ In addition to being one of the leading causes of hospitalization, disparities in the burden of heart failure have been identified, with studies reporting poorer quality of life, more frequent hospitalizations, and decreased survival among African-Americans and Hispanics relative to Caucasians. ${ }^{14-16}$

Recent studies have found that a growing number of HF cases are being reported in patients with current or prior use of methamphetamine. Methamphetamineinduced cardiomyopathy or HF seems to strike younger patients, racial/ethnic minorities and in some case reports, were found to be reversible. 6, 8, 9, 12,17-19 Yeo and colleagues conducted a case-control study $(\mathrm{N}=221)$ on heart failure patients admitted to a tertiary care hospital in Hawai' $i$ and found that methamphetamine users $(n=107)$ had a 3.7-fold higher odds ratio for cardiomyopathy after adjustment for age, BMI, and renal failure. ${ }^{9}$ Other basic science studies also have suggested that methamphetamine exposure is associated with structural and functional changes of myocytes resulting in cardiomyopathy and the clinical syndrome of congestive

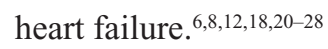

NHs and other PIPs are known to be at overall increased risk for heart disease as well as a number of risk factors for heart failure including diabetes, hypertension, tobacco use, and obesity ${ }^{13,14}$ and this population continues to experience disparities despite improvements in heart disease mortality experienced in the general US population. ${ }^{13,29}$ The purpose of this study is to examine socio-demographic and clinical risk factors associated with methamphetamine use in NHs and PIPs with moderate to severe heart failure.

\section{Methods}

Participants included in this study were enrolled in the Malama Pu'uwai (Heart Health Care) Study after informed consent was obtained. The Malama Pu'uwai Sudy (MPS) is a randomized controlled trial that is testing whether a culturally tailored educational program delivered post-discharge from the hospital will reduce re-hospitalization and/or mortality in NH and PIP patients with moderate to severe heart failure. All participants included in this study met the following eligibility criteria: 1) Self-identified as Native Hawaiian and/or Pacific Island racial/ethnic background (including Samoan, Tongan, Micronesian); 2) Age $\geq 21$ years; 3) Recently discharged from a single tertiary care hospital with a primary or secondary diagnosis of heart failure (index hospitalization); 4) moderate to severe heart failure defined as a left ventricular systolic ejection fraction (EF) $\leq 45 \%$ by echocardiography or similar cardiovascular imaging technique (eg, nuclear imaging, cardiac catheterization).

All participants underwent a baseline visit that included clinical assessment and a personal interview. Use of methamphetamine was determined by medical record review of the index hospitalization using a standardized protocol performed by a single trained reviewer or by a personal interview using standardized questionnaires at study entry. Positive methamphetamine use (MU) was defined as written documentation by a health care professional (eg, nurse, physician, social worker) or positive toxicology screen or if patient reported a history of methamphetamine use by interview. Participants without documentation of MU were categorized as non-methamphetamine users (non-MU) and served as the comparison group.

A total of 82 participants were enrolled between June 1, 2006 and December 31, 2007 in the MPS and were included in this study. All participants were interviewed and evaluated per the MPS protocol for: 1) demographics (ie, age, race/ethnicity - participants were allowed to selfidentify multiple race/ethnicity groups and then asked to select the group they identified the most with, marital status and education); 2) assessment of alcohol consumption in the past 12 months (yes or no); and 3) smoked 100 or more cigarettes in their lifetime (yes or no); 4) Medication use, including prescription and/or non-prescription; 5) Clinical assessment of blood pressure, pulse, weight and measured height using standardized protocols. Medical records 
from the index hospitalization also were reviewed for the following co-morbid conditions: 1) hypertension - defined as blood pressure $\geq 140 / 90 \mathrm{mmHg}$ or known history of hypertension; 2) diabetes - defined as known history of diabetes by a medical professional; 3) atherosclerotic heart disease - defined as history of myocardial infarction, coronary bypass surgery or angioplasty or other percutaneous revascularization procedure; 4) history of cerebrovascular disease or stroke.

\section{Statistics}

Odds ratios were used to measure the association of risk factors between MU and non-MU and were computed using unconditional logistic regression. ${ }^{15}$ The $95 \%$ confidence intervals for the OR estimates were determined using normal theory approximation. Significance testing of regression coefficients was based on Wald's statistic. Any univariate factors associated with methamphetamine use was included into subsequent logistic regression modeling if the score statistic for that variable was statistically significant after adjustment for multiplicity using the Hochberg procedure. ${ }^{16}$ The Fisher's exact test was used for comparison of categorical variables and Student's t-test for continuous variables. A p-value of $<0.05$ was considered to be statistically significant. Statistical analysis was performed using SAS statistical software version 9.1.3 (SAS Institute, Cary, NC).

\section{Results}

Current or prior history of MU occurred in $22 \%$ of Native Hawaiians (NHs) and Pacific Island Peoples (PIPs) with moderate to severe heart failure. MUs were significantly younger (mean age MU 43 years vs non-MU 58 years.; $\mathrm{p}=<0.0001$ ), more likely to be NHs than PIPs and were more likely to be non-married (never married $\mathrm{OR}=13,95 \% \mathrm{CI}$, 2.6-68; divorced/separated OR $=10,95 \% \mathrm{CI}, 1.7-62)$. MU was associated with higher BMI (BMI $\geq 30-39 \mathrm{~kg} / \mathrm{m}^{2}$, OR 4.9; BMI $\geq 39 \mathrm{~kg} / \mathrm{m}^{2}$, OR 3.9), higher pulse (OR 2.3-3.4), being a smoker (OR 2.9), an alcohol user (OR 2.2), or having a college degree (OR 2.8). However, none of these trends reached statistical significance. No significant differences (all odds ratios $\leq 1.9$ ) were observed between MU and non-MU with regard to sex, or systolic or diastolic blood pressure (Tables 1 and 2).

Table 2 shows that the distribution of co-morbid conditions was similar between MU and non-MU. The most frequent co-morbid condition was hypertension (68\%), followed by atherosclerotic heart disease (ASHD) at $62 \%$ and diabetes at $45 \%$. The occurrence of ASHD was more frequent in the non-MU (67\%) participants compared with the MU (44\%), but was not statistically significant. Overall, both the MU and the non-MU had an equal frequency of underlying co-morbid conditions despite the fact that the MU group was significantly younger.

Using multiple logistic regression adjusting for all relevant variables, we found that older age $(\geq 50$ years old) was a 'protective' risk factor for $\mathrm{MU}(\mathrm{OR}=0.16$, 95\% CI, 0.03-0.84) compared with non-MU. Non-married status (never married or divorced/separated) also was independently associated with an increased risk for MU (never married OR $=8.5,95 \% \mathrm{CI}, 1.5-47$; divorced or separated OR $=11,95 \% \mathrm{CI}, 1.8-75)($ Table 3). After adjustment for multiplicity, race/ethnicity was no longer a statistically significant predictor of MU and consequently was not included in the multiple logistic regression model. Subsequent regression models were analyzed sequentially adding the following covariates: race/ethnicity, sex, education, alcohol use, smoking, atherosclerotic heart disease, hypertension, diabetes, and stroke. However, none of these models changed the independent association of age and marital status with MU. Thus, we report in Table 3 the independent association of younger age and non-married status with positive MU compared with non-MUs in a population of NHs and PIPs with moderate to severe HF.

\section{Discussion}

Methamphetamine use occurs frequently (22\%) among $\mathrm{NH}$ and PIP patients with moderate to severe (LVEF $\leq 45 \%)$ heart failure. This finding adds to the growing clinical recognition of methamphetamine use in heart failure patients and in particular among high risk populations with cardiovascular disparities. ${ }^{30}$ In a study by Yeo et al $43 \%$ of patients with cardiomyopathy were found to be MUs (prior or current users) and the majority of patients with cardiomyopathy and MU were NHs and PIPs (54\%). ${ }^{9}$ Studies among primarily Caucasian patients also have reported nearly $20 \%$ of HF patients having a prior history of alcohol and/or substance abuse in addition to other co-morbid conditions, such as hypertension and diabetes. ${ }^{31}$ Our study is consistent with these findings of more than $20 \%$ of patients with heart failure having MU as a contributing cause. In this high risk patient population, we found that methamphetamine users were significantly more likely to be NHs compared with PIPs. Thus, although hypertension, ASHD, and diabetes are frequent underlying causes of $\mathrm{HF}$, other potential cardiac insults such as methamphetamine use may pose additional cardiotoxicity. ${ }^{30}$ 
Table I Demographic characteristics of Native Hawaiian and Pacific Island people with moderate to severe heart failure by methamphetamine use $(\mathrm{N}=82)$

\begin{tabular}{|c|c|c|c|}
\hline Variable & $\begin{array}{l}\text { Positive methamphetamine } \\
\text { use }(n=18) n(\%)\end{array}$ & $\begin{array}{l}\text { Negative methamphetamine } \\
\text { use }(n=64) n(\%)\end{array}$ & Odds ratio $(95 \% \mathrm{Cl})$ \\
\hline \multicolumn{4}{|l|}{ Age } \\
\hline$<40$ years & $6(33)$ & $6(9)$ & I.0 Referent \\
\hline $40-49$ years & $6(33)$ & $10(16)$ & $0.60(0.13-2.7)$ \\
\hline$\geq 50$ years & $6(33)$ & $48(75)$ & $0.13(0.03-0.51)$ \\
\hline \multicolumn{4}{|l|}{ Sex } \\
\hline Female & $4(22)$ & $22(34)$ & I.0 Referent \\
\hline Male & $14(78)$ & $42(66)$ & I.8 (0.54-6.2) \\
\hline \multicolumn{4}{|l|}{ Marital status } \\
\hline Currently married & $2(I I)$ & $37(58)$ & I.0 Referent \\
\hline Never & $10(56)$ & $14(22)$ & $13(2.6-68)$ \\
\hline Divorced or separated & $5(28)$ & $9(14)$ & $10(1.7-62)$ \\
\hline Widow or widower & I (6) & $4(6)$ & $4.6(0.34-63)$ \\
\hline \multicolumn{4}{|l|}{ Education level } \\
\hline Less than high school & $4(22)$ & $10(16)$ & I.0 Referent \\
\hline High school diploma/GED & $9(50)$ & $32(5 \mathrm{I})$ & $0.77(0.20-3.0)$ \\
\hline Some college/technical training & $2(I I)$ & $18(29)$ & $0.31(0.05-2.0)$ \\
\hline College degree & $3(17)$ & $3(5)$ & $2.8(0.39-20)$ \\
\hline \multicolumn{4}{|l|}{ Race/Ethnicity } \\
\hline Native Hawaiian & $16(89)$ & $40(63)$ & I.0 Referent \\
\hline Pacific Island people & $2(I I)$ & $24(38)$ & $0.21(0.04-.99)$ \\
\hline \multicolumn{4}{|l|}{ Smoked 100 cigarettes in lifetime (\%) } \\
\hline No & $2(I I)$ & $17(27)$ & I.0 Referent \\
\hline Yes & $16(89)$ & $47(73)$ & $2.9(0.60-14)$ \\
\hline \multicolumn{4}{|l|}{ Alcohol consumed in past I 2 months } \\
\hline No & $5(28)$ & $29(45)$ & I.0 Referent \\
\hline Yes & $13(72)$ & $35(55)$ & $2.2(0.68-.8)$ \\
\hline
\end{tabular}

Our results also suggest that among racial/ethnic populations at high risk for cardiovascular disparities, such as heart failure, consideration of alterNative causes of HF may be important contributors to the manifestation of CVD disparities. Racial/ethnic differences in HF risk factors also was examined by Deswal et al in Black patients with advanced HF in a VA system. ${ }^{32}$ The authors found that uncontrolled hypertension and hospitalization for any cause accounted for a significant difference in health disparities when compared with their White counterparts. ${ }^{32}$ Similarly, our results suggest that the frequency or severity of known underlying causes of HF, such as MU in addition to a background of hypertension, ASHD and diabetes, may have a significant additive effect on the development of CVD outcomes especially among NHs and PIPs. Surprisingly little is known about the prevalence of methamphetamine use in patients with heart failure. Yet it is well known that racial and ethnic minority patients have persistent disparities on the severity and treatment of heart failure that may contribute to differences in health outcomes. Miller recently highlighted the gap in our fund of knowledge between heart failure patients that have been included in clinical trials that guide practice guidelines and heart failure patients that are racially and demographically more diverse and for whom the treatment guidelines may not be optimal treatment. Thus, our studies provides new insight to the clinical and demographic characteristics of NHs and PIPs who are at increased risk for heart failure and for poor health outcomes. ${ }^{33}$

Our study is the first to identify not only the increased frequency of MU among NH and PIP HF patients but also to examine the potential risk factors associated with $\mathrm{MU}$ in this distinct population. Although MU has been recognized as a growing health problem in the US since the early 1990s, little is available in the literature about its long term impact on 
Table 2 Clinical characteristics among Native Hawaiian and Pacific Island peoples with moderate - severe heart failure by methamphetamine use $(\mathrm{N}=82)$

\begin{tabular}{|c|c|c|c|}
\hline Clinical characteristic & $\begin{array}{l}\text { Positive methamphetamine } \\
\text { use }(n=18) n(\%)\end{array}$ & $\begin{array}{l}\text { Negative methamphetamine } \\
\text { use }(n=64) n(\%)\end{array}$ & Odds ratio $(95 \% \mathrm{Cl})$ \\
\hline \multicolumn{4}{|l|}{ Blood pressure } \\
\hline \multicolumn{4}{|l|}{ Systolic blood pressure* } \\
\hline$<140 \mathrm{mmHg}$ & $16(89)$ & $60(94)$ & I.0 Referent \\
\hline$\geq 140 \mathrm{mmHg}$ & $2(11)$ & $4(6)$ & $1.9(0.32-1 \mathrm{I})$ \\
\hline \multicolumn{4}{|l|}{ Diastolic blood pressure* } \\
\hline$<90 \mathrm{mmHg}$ & $16(89)$ & $59(92)$ & I.0 Referent \\
\hline$\geq 90 \mathrm{mmHg}$ & $2(11)$ & $5(8)$ & I.5 (0.26-8.3) \\
\hline \multicolumn{4}{|l|}{ Pulse* } \\
\hline$<65$ beats per minute & $3(17)$ & $22(34)$ & I.0 Referent \\
\hline$>65$ to 85 beats per minute & $9(50)$ & $29(45)$ & $2.3(0.55-9.4)$ \\
\hline$\geq 85$ beats per minute & $6(33)$ & $13(20)$ & $3.4(0.72-16)$ \\
\hline \multicolumn{4}{|l|}{ Body mass index } \\
\hline$<30 \mathrm{~kg} / \mathrm{m}^{2}$ & $2(11)$ & $22(34)$ & I.0 Referent \\
\hline$\geq 30$ to $39 \mathrm{~kg} / \mathrm{m}^{2}$ & $8(44)$ & $18(28)$ & $4.9(0.92-26)$ \\
\hline$\geq 39 \mathrm{~kg} / \mathrm{m}^{2}$ & $6(33)$ & $17(27)$ & $3.9(0.70-22)$ \\
\hline Unknown & $2(11)$ & $7(11)$ & $3.1(0.37-27)$ \\
\hline \multicolumn{4}{|l|}{ Systolic Ejection Fraction, \% } \\
\hline Severe $(<30)$ & II (6I) & $36(56)$ & 1.0 Referent \\
\hline Moderate-severe (30-34) & $I(6)$ & $7(11)$ & $0.47(0.05-4.2)$ \\
\hline Moderate (35-39) & $6(33)$ & $11(17)$ & I.8 (0.54-5.9) \\
\hline Mild-Moderate (40-44) & $0(0)$ & $10(16)$ & $0.16(0.0088-3.0)^{\dagger}$ \\
\hline \multicolumn{4}{|l|}{ Atherosclerotic Heart Disease } \\
\hline No & $10(56)$ & $21(33)$ & I.0 Referent \\
\hline Yes & $8(44)$ & $43(67)$ & $0.39(0.14-1.1)$ \\
\hline \multicolumn{4}{|l|}{ Stroke* } \\
\hline No & $16(89)$ & $58(91)$ & I.0 Referent \\
\hline Yes & $2(11)$ & $6(9)$ & I.2 (.22-6.6) \\
\hline \multicolumn{4}{|l|}{ Diabetes mellitus* } \\
\hline No & $10(56)$ & $35(55)$ & I.0 Referent \\
\hline Yes & $8(44)$ & $29(45)$ & $0.97(0.34-2.8)$ \\
\hline \multicolumn{4}{|l|}{ Hypertensiona* } \\
\hline No & $5(28)$ & $21(33)$ & I.0 Referent \\
\hline Yes & $13(72)$ & $43(67)$ & I.3 (0.40-4.0) \\
\hline
\end{tabular}

*Missing values $(\leq 2)$ were absorbed into the referent groups.

tMissing cell imputed with 0.5 in order to compute OR and $\mathrm{Cl}$.

heart failure specifically and/or its impact on the development of cardiovascular disease in general.

A few studies have examined the differences in patient related factors among minority patients with heart failure compared with their white counter parts. Deswal et al found in a VA population that non-marital status was higher in Black patients with HF compared with Whites. ${ }^{32}$ Our study, in another high risk minority population, is in agreement with this study and others that suggests that greater awareness of psychosocial factors and MU in HF patients is needed and may have important implications for successful chronic disease management. In particular, programs aimed at treating and/or preventing HF hospitalizations and death in health disparate populations should consider the possibility of psychosocial issues (eg, marital status, social support) in patients with heart failure and a prior or current history of 
Table 3 Multivariate analysis of factors associated with methamphetamine use in Native Hawaiians and Pacific Island peoples with moderate to severe heart failure $(\mathrm{N}=82)^{*}$

\begin{tabular}{ll}
\hline Factors & Odds ratio $(95 \% \mathrm{Cl})$ \\
\hline $\begin{array}{l}\text { Age (years) } \\
<40\end{array}$ & I.0 Referent \\
$\geq 40$ to 50 & $0.70(0.13-3.9)$ \\
$>50$ & $0.16(0.03-0.84)$ \\
Marital status & \\
Currently married & I.0 Referent \\
Never married & $8.5(1.5-47)$ \\
Divorced or separated & II $(1.8-75)$ \\
Widow or widower & $5.7(0.38-87)$ \\
\hline
\end{tabular}

*Adjustment for race/ethnicity, sex, education, alcohol use, smoking, atherosclerotic heart disease, stroke, hypertension, and diabetes were not significant confounders to the model.

MU as they relate to successful heart failure management. Specifically, patients with HF that have a prior history of MU may be younger than the typical HF patient and may have a need for alterNative approaches to ensure adherence to the treatment regimen.

Other studies have examined the role of marital status on health outcomes in HF patients. One study found that among HF patients that were married, the quality of this relationship independently predicted survival, especially among women HF patients. ${ }^{34}$ Another study found that high social networking, irrespective of marital status, reduced re-hospitalization in HF patients. ${ }^{35}$ Our results support the 'protective' role of being married on the association of MU in HF patients. The mechanism for how being married may 'protect' against MU has been proposed to be via social support that may mitigate the risk for illicit drug use. This proposed mechanism is supported by other studies in which patients with heart failure who were unmarried had a lower level of self-care knowledge and adherence than those who were married, indicating that marriage improves the ability to care for one's self. ${ }^{36}$ The long term benefits of social support in HF patients is consistent with a wealth of literature that has reported that improved health status and social support are associated with decreased hospitalizations or all cause death. ${ }^{37}$

Unfortunately, the cross sectional design of our study does not allow for determining cause and effect relationships between marital status and MU in HF patients. However, recognition of MU in HF patients is important not only for diagnostic purposes but also for long term treatment and prevention of HF in patients from minority or health disparate populations. Further delineation of which factors (ie, hypertension, diabetes, methamphetamine, etc.) either collectively or individually can be attributed to the development of heart failure in this population will aid our understanding in developing appropriate treatments to reverse these trends. Our study also highlights an opportunity to intervene early to prevent advanced heart failure in $\mathrm{NH}$ and PIP patients with a prior history of MU who may benefit from programs that can provide a supportive context to prevent relapse of MU as well as HF disease management. Further understanding as to the underlying risk factors leading to $\mathrm{HF}$ are needed in health disparity populations. Innovative approaches to reduce and/or prevent the development of advanced HF in these populations has important social and economic implications for the health burden of HF in the US since HF is one of the most costly disease hospitalizations in the US..$^{38,39}$

Several limitations should be considered when interpreting the results of this study. The cross-sectional design precludes making any causal inferences between the model risk factors and MU. While the limited sample size may be contributing to the lack of statistical significance observed for some of the risk factors examined. Medical record review of methamphetamine use may also underestimate the actual number of methamphetamine users and other illicit drugs such as cocaine were not assessed in this study. Despite these limitations, this study provides initial findings to a growing body of literature that is recognizing with greater frequency the role of methamphetamine use as a significant contributor to the development of HF. Because MU and heart failure tends to occur more frequently in racial and ethnic minority populations and originally became manifest in the western regions of the US where the majority of NHs and PIPs reside. This may be, just the beginning of a larger, but as of yet unrecognized, problem that contributes to the management of heart failure and cardiomyopathy especially in high risk minority or ethnic populations.

Our study suggests that MU in NHs and PIPs with moderate to severe heart failure occurs at a frequency of more than 1 in 5 cases (22\%). Factors independently associated with MU in heart failure patients of NHs and PIP ancestry were non-marital status and younger age. Intervention efforts to reduce persistent cardiovascular health disparities, specifically heart failure, may want to consider strategies to also reduce MU in this and other high risk patients. Further studies are needed to identify key modifiable risk factors that may enable better health outcomes in health disparate populations (eg, NHs and other PIPs) if we hope to reverse and eventually eliminate heart failure disparities. 


\section{Acknowledgments}

We acknowledge the contributions to the staff of the Malama Pu'uwai Study: Dr J. Keawe'aimoku Kaholokula, PhD; Nobumi Nakamura, Sharmaine Sylva, Theresa Sawchuck, and Jo Ann Kimura, L. Ka'ohimanu Dang from the Department of Native Hawaiian Department at the John A. Burns School of Medicine and May Vawer at the Queens Medical Center for their support and resources. This study received funding support from the following grants: U01 HL079163 (UH Partner - Heart Failure Disparities in Native Hawaiians), U01 HL079152 (QMC Partner - Heart Failure Disparities in Native Hawaiians); P20 MD 000173 (Hawaii EXPORT Center). The contents of this publication are solely the responsibility of the authors and do not necessarily represent the official views of the funding agencies.

\section{Disclosures}

None of the authors have conflicts of interest to disclose.

\section{References}

1. NIDA Research Report. Methamphetamine abuse and addiction: NIH Publication No 06-4210: NIDA Research Report; 2006.

2. Substance Abuse and Mental Health Services Administration. Results from the 2005 National Survey on Drug Use and Health: National Findings Rockville, MD: Office of Applied Studies, NSDUH Series H-30, DHHS Publication No. SMA 06-4194; 2006.

3. Alcohol and Drug Abuse Division, Hawaii Department of Health. Substance abuse in Hawaii: adult population household telephone survey. Kapolei, HI; 1998.

4. Alcohol and Drug Abuse Division, Hawaii Department of Health. Substance abuse and treatment needs: survey estimates for Hawai' $i$. Kapolei, HI; 1998.

5. Alcohol and Drug Abuse Division, Hawaii Department of Health. $\mathrm{Ka}$ leo o na keiki: the 2003 Hawaii student alcohol, tobacco, and other drug use study (1987-2003). Kapolei, HI; 2003.

6. Hong R, Matsuyama E, Nur K. Cardiomyopathy associated with the smoking of crystal methamphetamine. JAMA. 1991;265(9):1152-4.

7. Inouye DS, Navin JJ, Hardman JM. Fatal postoperative arrhythmia in a man with a remote history of methamphetamine and cocaine use: a case report. Hawaii Med J. 2004;63(3):82-6.

8. Wijetunga M, Seto T, Lindsay J, Schatz I. Crystal methamphetamineassociated cardiomyopathy: tip of the iceberg? J Toxicol Clin Toxicol 2003;41(7):981-6.

9. Yeo KK, Wijetunga $\mathrm{M}$, Ito $\mathrm{H}$, et al. The association of methamphetamine use and cardiomyopathy in young patients. Am J Med. 2007;120(2):165-71.

10. Substance Abuse and Mental Health Services Administration, Office of Applied Studies. Drug Abuse Warning Network, 2004. National Estimates of Drug-Related Emergency Department Visits. Rockville, MD: DAWN Series D-28, DHHS Publication No. (SMA) 06-41432006.

11. Substance Abuse and Mental Health Services Administration, Office of Applied Studies. Drug Abuse Warning Network, 2005: National Estimates of Drug-Related Emergency Department Visits. Rockville, MD DAWN Series D-29, DHHS Publication No. (SMA) 07-4256;2007.

12. Jacobs LJ. Reversible dilated cardiomyopathy induced by methamphetamine. Clin Cardiol. 1989;12(12):725-7.

13. American Heart Association. Heart disease and stroke statistics - 2007 update. Dallas, Texas: American Heart Association; 2007.
14. Bosworth HB, Steinhauser KE, Orr M, Lindquist JH, Grambow SC, Oddone EZ. Congestive heart failure patients' perceptions of quality of life: the integration of physical and psychosocial factors. Aging Ment Health. 2004;8(1):83-91.

15. Jerant AF, von Friederichs-Fitzwater MM, Moore M. Patients' perceived barriers to active self-management of chronic conditions. Patient Educ Couns. 2005;57(3):300-7.

16. Yancy $\mathrm{CW}$. The prevention of heart failure in minority communities and discrepancies in health care delivery systems. Med Clin North Am. 2004;88(5):1347-1368, xii-xiii.

17. Chen YT, Vaccarino V, Williams CS, Butler J, Berkman LF, Krumholz HM. Risk factors for heart failure in the elderly: a prospective community-based study. Am J Medicine. 1999;106(6):605-12.

18. He SY, Matoba R, Fujitani N, Sodesaki K, Onishi S. Cardiac muscle lesions associated with chronic administration of methamphetamine in rats. Am J Forensic Med Pathol. 1996;17(2):155-62.

19. Varner KJ, Ogden BA, Delcarpio J, Meleg-Smith S. Cardiovascular responses elicited by the "binge" administration of methamphetamine. J Pharmacol Exp Ther. 2002;301(1):152-9.

20. Islam MN, Kuroki H, Hongcheng B, et al. Cardiac lesions and their reversibility after long term administration of methamphetamine. Forensic Sc Int. 1995;75(1):29-43.

21. Kaiho M, Ishiyama I. Morphological study of acute myocardial lesions experimentally induced by methamphetamine. Nihon Hoigaku Zasshi. 1989;43(6):460-8.

22. Kalant H, Kalant OJ. Death in amphetamine users: causes and rates. Can Med Assoc J. 1975;112(3):299-304.

23. Karch SB, Stephens BG, Ho CH. Methamphetamine-related deaths in San Francisco: demographic, pathologic, and toxicologic profiles. J Forensic Sci. 1999;44(2):359-68.

24. Logan BK, Fligner CL, Haddix T. Cause and manner of death in fatalities involving methamphetamine. J Forensic Sci. 1998;43(1):28-34.

25. Maeno Y, Iwasa M, Inoue H, Koyama H, Matoba R, Nagao M. Direct effects of methamphetamine on hypertrophy and microtubules in cultured adult rat ventricular myocytes. Forensic Sci Int. 2000;113(1-3):239-43.

26. Maruta T, Nihira M, Tomita Y. [Histopathological study on acute poisoning of methamphetamine, morphine or cocaine]. Nihon Arukoru Yakubutsu Igakkai Zasshi. 1997;32(2):122-38.

27. Robinson M, Turnipseed S, Glatter K. Methamphetamine-associated cardiomyopathy: a previously unrecognized cause of heart failure. American Heart Association Annual Scientific Sessions. New Orleans; 2004.

28. Smith HJ, Roche AH, Jausch MF, Herdson PB. Cardiomyopathy associated with amphetamine administration. Am Heart J. 1976;91(6):792-7.

29. Grandinetti A, Chang HK, Mau MK, et al. Prevalence of glucose intolerance among Native Hawaiians in two rural communities. Native Hawaiian Health Research (NHHR) Project. Diabetes Care. 1998;21(4):549-54.

30. Vasan RS, Wilson PW. Epidemiology and causes of heart failure. UpToDate Online 15.3. Accessed November 28, 2007. Available from www.uptodate.com.

31. Rumsfeld JS, Havranek E, Masoudi FA, et al. Depressive symptoms are the strongest predictors of short-term declines in health status in patients with heart failure. J Am Coll Cardiol. 2003;42(10):1811-7.

32. Deswal A, Petersen NJ, Urbauer DL, Wright SM, Beyth R. Racial variations in quality of care and outcomes in an ambulatory heart failure cohort. Am Heart J. 2006;152(2):348-54.

33. Miller LW. Heart Failure: Who We Treat Versus Who We Study. Cardiol Clin. 2008;26:113-25.

34. Rohrbaugh MJ, Shoham V, Coyne JC. Effect of marital quality on eight-year survival of patients with heart failure. Am J Cardiol. 2006;98(8):1069-72.

35. Rodriguez-Artalejo F, Guallar-Castillon P, Herrera MC, et al. Social network as a predictor of hospital readmission and mortality among older patients with heart failure. Journal of Cardiac Failure. Oct 2006;12(8):621-7. 
36. Ni H, Nauman D, Burgess D, Wise K, Crispell K, Hershberger RE. Factors influencing knowledge of and adherence to self-care among patients with heart failure. Arch Int Med. 1999;159(14):1613-9.

37. Heidenreich PA, Spertus JA, Jones PG, et al. Health status identifies heart failure outpatients at risk for hospitalization or death. J Am Coll Cardiol. 2006;47(4):752-6.
38. Gottlieb SS, Mann DL, Francis GS. Highlights of the 2006 scientific sessions of the Heart Failure Society of America: Seattle, Washington, September 10-13, 2006. J Am Coll Cardiol. 2007;49(5):608-15.

39. Rich MW, Nease RF. Cost-effectiveness analysis in clinical practice: the case of heart failure. Arch Int Med. 1999;159(15):1690-700. 